(C)Auteurs. Cette œuvre, disponible à http://dx.doi.org/10.18162/fp.2020.677, est distribuée sous licence Creative Commons Attribution 4.0 International http://creativecommons.org/licences/by/4.0/deed.fr

\section{Intégrer les contraintes de la pandémie dans les analyses préalables, la conception et l'expérimentation d'ingénieries didactiques en éducation physique. Un exemple en course d'orientation}

\author{
Integrating pandemic constraints into the preliminary analysis, design, \\ and testing of didactic engineering for physical education. \\ An example of an orienteering course
}

\section{ésumé}

Cet article rend compte d'une démarche d'ingénierie didactique pour le développement et la formation visant à assurer la continuité pédagogique en éducation physique dans le contexte de la pandémie COVID-19. À l'aide d'un exemple en course d'orientation, nous montrons le double effet des contraintes de la pandémie sur la démarche d'ingénierie : (a) celui de catalyseur pour la conception de séquences didactiques intégrant les MITIC et préservant les enjeux d'apprentissage et de transmission culturelle de l'éducation physique; (b) celui d'obstacle restreignant l'accès au terrain scolaire pour expérimenter et valider les séquences produites.

Mots-clés

Éducation physique, ingénierie didactique, course d'orientation, pandémie, $\mathrm{TIC}$

Abstract

This article describes a didactic engineering approach to development and training with a view to ensuring learning continuity in physical education during the COVID-19 pandemic. Through an example of an orienteering course, we demonstrate the dual effect of pandemic constraints on the engineering approach: (a) as a catalyst for the design of didactic sequences that integrate media, images, and ICT to sustain educational games and the cultural transmission of physical education; and (b) as a barrier to school access for testing and validating the produced sequences.

Keywords

Physical education, didactic engineering, orienteering course, pandemic, ICT

\section{Introduction}

Durant la période de pandémie et de semi-confinement qu'a connue la Suisse romande entre mars et mai 2020, assurer la continuité pédagogique a constitué l'une des préoccupations majeures de la communauté éducative. En éducation physique (EP), cela a représenté un véritable défi pour le corps enseignant. De nombreuses propositions de contenus sont rapidement apparues sur les réseaux sociaux et sur le Web, à l'initiative d'associations professionnelles, d'enseignants d'EP, d'instructeurs de fitness, etc. Ces propositions comportaient majoritairement les caractéristiques suivantes: (a) elles se focalisaient exclusivement sur les dimensions fonctionnelles et hygiénistes, au détriment des enjeux d'apprentissage et de transmission culturelle de l'EP (Forest, Lenzen et Öhman, 2018); (b) elles se présentaient sous la forme de tâches isolées, en rupture avec la logique de séquence didactique ou de projet d'enseignement sur laquelle se fonde l'enseignement de l'EP comme celui des autres disciplines scolaires; (c) elles prescrivaient un travail individuel aux élèves, à l'encontre de la dimension éminemment collective de l'EP à travers les notions d'équipes, de rôles sociaux, etc. (Travert et Mascret, 2011). Devant ce constat, dans l'urgence qui prévalait durant cette période, un groupe de recherche en didactique de l'EP de l'Université de Genève a adopté une démarche d'ingénierie didactique visant à élaborer des contenus dans différentes activités physiques, sportives et artistiques (APSA), à mettre ceux-ci à disposition de la communauté éducative sur le site Web du groupe, et à étudier leur implémentation lorsque cela était possible. Dans cette contribution, nous rendons compte de cette démarche à travers une proposition curriculaire en course d'orientation $(\mathrm{CO})$, qui a été mise à l'épreuve dans le programme de formation des enseignants d'une haute école pédagogique partenaire. Nous mettons plus spécifiquement l'accent sur les contraintes de la 
pandémie, qui ont joué un rôle déterminant à la fois dans le traitement didactique de la pratique sociale de référence (Martinand, 2001) et dans l'expérimentation du produit de ce traitement didactique.

\section{Cadre théorique et méthodologique}

La notion d'ingénierie didactique (ID) a émergé en didactique des mathématiques au début des années 1980, en réponse à deux questions cruciales de l'époque (Artigue, 1988) : (a) les rapports entre la recherche et l'action sur le système d'enseignement; et (b) le rôle qu'il convient de faire jouer aux réalisations didactiques en classe, au sein des méthodologies de la recherche didactique. Depuis lors, l'ID a été étendue à d'autres champs disciplinaires, dont l'EP (Amade-Escot et Marsenach, 1995; Carnus, Sauvegrain et Terrisse, 2002), avec la même visée de transformation du système didactique par la remise en cause des contenus enseignés et la construction de contenus rénovés (Marsenach et Amade-Escot, 1993).

Plus récemment, Perrin-Glorian $(2011,2019)$ a distingué de nouvelles formes d'ID, parmi lesquelles l'ingénierie didactique pour le développement et la formation (IDD). Celle-ci reste une méthodologie de recherche, mais prend explicitement en compte la question de la diffusion dans l'enseignement des situations élaborées, les premiers travaux d'ID ayant montré que la transmission des produits de l'ID nétait pas évidente (Artigue et Perrin-Glorian, 1991). L'IDD adopte le découpage temporel classique de la méthodologie d'ingénierie, à savoir (Artigue, 1988; Perrin-Glorian, 2019) :

1. Les analyses préalables. Cette " analyse épistémologique et didactique qui précède nécessairement la construction d'ingénieries didactiques »(Charnay, 2003, p. 19) porte notamment sur les contenus visés par l'enseignement, leur place et leur rôle dans la discipline scolaire concernée, les conceptions des élèves, les difficultés et obstacles qui marquent leur évolution, le champ de contraintes dans lequel va se situer la réalisation didactique effective.

2. La conception et l'analyse a priori. La phase de conception de séquences ou contenus rénovés « associe [dans certains cas] des enseignants et des mises en œuvre tatonnées dans les conditions normales d'enseignement » (Marsenach et Amade-Escot, 1993, p. 36). Elle s'accompagne d'une analyse a priori, conçue comme une analyse du contrôle du sens dont l'objectif est de déterminer en quoi les choix effectués lors de la conception permettent de contrôler les comportements des élèves et leur sens (Artigue, 1988). Il s'agit d'un travail d'hypothèses sur les démarches, stratégies, raisonnements que l'élève peut mettre en œuvre compte tenu de ses ressources du moment, sur les difficultés qu'il peut rencontrer et les erreurs qu'il peut commettre, sur l'effet des variables didactiques et pédagogiques sur son travail (Charnay, 2003).

3. L'expérimentation. Des données sont collectées lors de l'implémentation en classe et l'observation de la séquence ou des contenus élaborés à l'étape précédente.

4. L'analyse a posteriori de cette séquence ou de ces contenus et la confrontation à l'analyse a priori. Cette phase s'appuie sur l'ensemble des données recueillies lors de l'expérimentation (observation des séances d'enseignement, productions des élèves en classe ou hors classe), auxquelles peuvent s'ajouter des données obtenues par l'utilisation de méthodologies externes (questionnaires, entretiens).

Nous faisons le choix de structurer la partie empirique de ce texte selon ces quatre phases, en mêlant

2 - Formation et profession 28(4 hors-série), 2020 
au sein de chacune de ces sections, si cela est approprié, des aspects relevant classiquement de la méthodologie et des résultats. Nous terminerons plus traditionnellement ce texte par une discussion et une conclusion.

\section{Partie empirique}

\section{Les analyses préalables}

APSA d'origine scandinave, la CO peut être définie comme une course individuelle ou collective en milieu inconnu, dans laquelle l'orienteur doit trouver à l'aide d'une carte et d'une boussole des éléments significatifs indiqués sur la carte (postes) et matérialisés sur le terrain (balises) plus rapidement que ses adversaires pour gagner la course (Mottet et Saury, 2014, p. 41).

$\mathrm{Si}$, dans toutes les APSA, le pratiquant doit prendre en compte des informations pertinentes de l'environnement, la spécificité de la $\mathrm{CO}$ réside dans le fait que l'orienteur doit effectuer une lecture conjointe des informations présentes sur le terrain et de celles représentées sur la carte. Le problème fondamental pour ce dernier consiste à rendre congruentes les informations de la carte et celles du terrain pour se situer et s'orienter (Mottet et Saury, 2014). Dans le cadre de l'enseignement de la $\mathrm{CO}$ en EP, le corps enseignant privilégie généralement l'utilisation de la carte, délaissant celui de la boussole. Le groupement le plus utilisé au niveau débutant est celui de la dyade d'élèves, favorisant les interactions entre les élèves et l'apprentissage d'un travail a priori coopératif. Les utilisations de la carte par les élèves et les interactions au sein de la dyade se déroulant rarement sous la supervision de l'enseignant (Jourand, Adé, Sève et Thouvarecq, 2016), cela force les élèves à faire preuve d'autonomie pour atteindre les objectifs fixés (Jourand, Adé, Sève, Komar et Thouvarecq, 2018).

Dans le système scolaire suisse romand, la CO fait partie des APSA associées à l'axe thématique «Pratiques sportives» du domaine disciplinaire «Corps et mouvement» du Plan d'études romand (PER) (Conférence Intercantonale de l'Instruction Publique de la Suisse Romande et du Tessin [CIIP], 2010-2016). Au cycle 3 de la scolarité obligatoire (élèves de 12 à 15 ans), elle est supposée concourir à l'objectif d'apprentissage « Entraîner des techniques et développer des habiletés motrices » et l'attente fondamentale ${ }^{1}$ qui lui est assignée est «Se déplace dans un espace en se repérant à l'aide d'une carte et/ou d'une boussole ». Dans le PER, les domaines disciplinaires se trouvent dans un rapport de complémentarité avec la formation générale et les capacités transversales. La première se structure en cinq thématiques déclinant différents aspects d'éducation et de transmission de valeurs (CIIP, 2010-2016). Au regard des contraintes de la pandémie et des spécificités de la CO, les concepteurs de l'IDD ont identifié deux thématiques plus susceptibles d'être prises en compte dans la phase de conception. Premièrement, il y a les MITIC (Médias, Images, Technologies de l'Information et de la Communication). Dans le contexte suisse romand où l'enseignement en présentiel était proscrit, mais où les déplacements neétaient pas limités à l'intérieur des cantons, les MITIC étaient ainsi appelés à jouer le double rôle d'objets d'apprentissage et d'outils permettant de médier les interactions entre l'enseignant et les élèves et entre les élèves. Deuxièmement, il y a les Choix et projets personnels. Cette thématique visant notamment à rendre l'élève autonome dans la gestion et la réalisation d'un projet (CIIP, 2010-2016), elle s'accordait particulièrement bien avec la CO (Jourand et al., 2018) et paraissait pouvoir être renforcée grâce à un traitement didactique spécifique de cette APSA. Par ailleurs, les 
capacités transversales représentent une part importante du bagage que chaque élève doit acquérir au cours de sa scolarité en vue de son insertion sociale et professionnelle. Au nombre de cinq, elles ne sont pas enseignées pour elles-mêmes, mais sont mobilisées au travers des situations d'apprentissage proposées par l'enseignant aux élèves (CIIP, 2010-2016). Dans le contexte de semi-confinement dans lequel a émergé l'IDD dont nous rendons compte ici, il a semblé à ses concepteurs que la Communication, « axée sur la mobilisation des informations et des ressources permettant de s'exprimer à l'aide de divers types de langages, en tenant compte du contexte »(CIIP, 2010-2016), constituait une capacité transversale qui rencontrait à la fois les caractéristiques de la $\mathrm{CO}$ et les besoins estimés des élèves. Celle-ci paraissait également pouvoir être renforcée grâce à un traitement didactique spécifique de cette APSA. Le tableau 1 synthétise les données institutionnelles issues des analyses préalables. Celles-ci ont déterminé une partie du cahier des charges que les concepteurs de l'IDD se sont donné dans la phase de conception.

\section{Tableau 1}

Données institutionnelles issues des analyses préalables

\begin{tabular}{|c|c|}
\hline Pratique sociale de référence & Course d'orientation \\
\hline Degré & Cycle 3 \\
\hline \multirow{9}{*}{ Plan d'études romand (PER) } & Objectif(s) d'apprentissage \\
\hline & CM 33 - Entraîner des techniques et développer des habiletés motrices \\
\hline & Attente(s) fondamentale(s) \\
\hline & $\begin{array}{l}\text { CM } 33 \text { - Se déplace dans un espace en se repérant à l'aide d'une carte et/ou d'une } \\
\text { boussole }\end{array}$ \\
\hline & Formation générale \\
\hline & MITIC \\
\hline & Choix et projets personnels \\
\hline & Capacités transversales \\
\hline & Communication \\
\hline
\end{tabular}

\section{La conception et l'analyse a priori}

Dans cette deuxième phase de l'IDD, ses concepteurs, en appui sur une conception élargie de la transposition didactique (Martinand, 2001; Perrenoud, 1998) et sur une tradition d'enseignement de l'EP comme éducation à la culture physique et sportive (Forest et al., 2018), se sont donné les règles de conduite suivantes:

- identifier, à partir des attentes fondamentales, des objectifs d'apprentissage et des pratiques 
sociales de référence du PER (cf. analyses préalables), les contenus d'enseignement les plus à même d'être transmis et acquis à distance;

- concevoir des séquences didactiques visant la transmission et l'acquisition de ces contenus, en faisant appel aux MITIC pour pallier l'absence de contact direct des élèves avec l'enseignant et leurs pairs.

Pour la $\mathrm{CO}$, il en a résulté une proposition curriculaire sous forme de duel, par dyades d'élèves habitant dans un même quartier ou village (tableau 2 ).

Tableau 2

Séquence didactique en course d'orientation

\begin{tabular}{|c|c|}
\hline Description du projet & $\begin{array}{l}\text { Course d'orientation sous forme de duel, par dyades d'élèves habitant dans un même quartier } \\
\text { ou village }\end{array}$ \\
\hline Contenus d'enseignement & $\begin{array}{ll}\text { - } & \text { Lecture de carte } \\
\text { - } & \text { Manipulation d'outils informatiques } \\
\text { - } & \text { Mémorisation de parcours } \\
\text { - } & \text { Gestion de la course } \\
\text { - } & \text { (Recherche d'informations sur Internet) }\end{array}$ \\
\hline $\begin{array}{l}\text { Aspects pratiques et } \\
\text { organisation pédagogique }\end{array}$ & 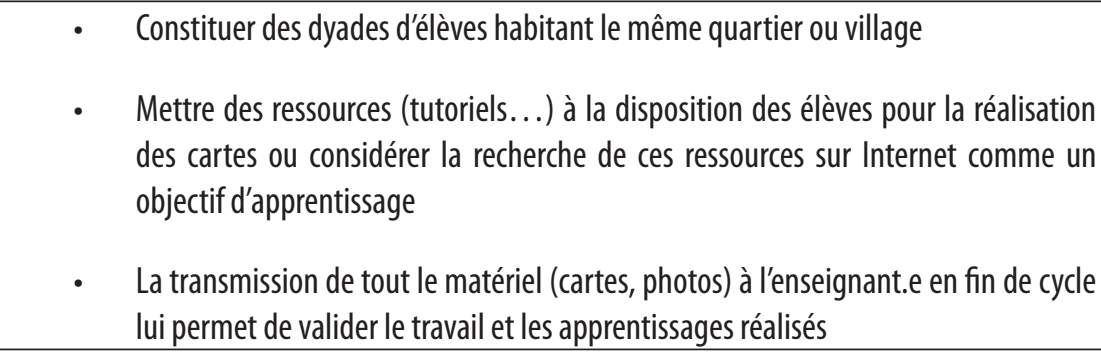 \\
\hline Matériel & $\begin{array}{l}\text { - Matériel informatique pour réaliser les cartes (ordinateur, tablette) } \\
\text { - Outil de communication numérique (ordinateur, tablette, smartphone) pour } \\
\text { échanger les documents au sein de la dyade } \\
\text { - Chronomètre }\end{array}$ \\
\hline
\end{tabular}


1. Constituer des dyades d'élèves habitant le même quartier ou village.

2. L'élève 1 prépare un itinéraire et le représente sur une carte (capture d'écran de Google Maps par exemple, ou carte numérique disponible gratuitement sur leWeb). II/elle marque un nombre défini de lieux (identifiables facilement) représentant les balises.

3. I//elle effectue le parcours le plus rapidement possible et à chaque balise/lieu remarquable, en prend une photo. Il/elle conserve son temps de course.

4. L'élève 1 transmet à l'élève 2 la carte avec le parcours et les balises représentés, ainsi que les photos des balises/lieux remarquables.

5. Lélève 2 effectue le parcours le plus rapidement possible et à chaque balise/lieu remarquable, prend un selfie. Il/elle conserve son temps de course.

6. Ensuite, inverser les rôles. L'addition des temps de course de chaque élève dans les deux situations (balisage du parcours et découverte du parcours) détermine le vainqueur de ce duel à distance.

7. Note: Il est important de sensibiliser les élèves (1) au respect des mesures de distance sociale en période de pandémie (la course se fait bien individuellement), et (2) au fair-play (I'usage de GPS est proscrit).

Cette séquence met en scène les trois rôles sociaux (Travert et Mascret, 2011) les plus emblématiques de la CO : le traceur, le poseur et l'orienteur (Swiss Orienteering, 2009-2010). Pour réaliser sa tâche, le traceur-poseur doit d'abord, en tant que traceur, utiliser les applications et/ou logiciels appropriés pour tracer un parcours sur une carte numérique. Il doit ensuite, en tant que poseur, rendre congruentes les informations de la carte et celles du terrain (Mottet et Saury, 2014) pour poser virtuellement les balises à l'endroit des postes représentés sur la carte (au moyen de photographies) ${ }^{2}$. Il doit enfin communiquer à l'orienteur les informations et matériaux nécessaires à la réalisation de sa course (dans le langage spécifique à la $\mathrm{CO}$, aidé du langage photographique permettant de surmonter les contraintes de la pandémie).

L'analyse a priori permet de pointer que, pour que la séquence fonctionne, les élèves doivent :

- mobiliser - ou acquérir via les ressources mises à leur disposition par l'enseignant - des habiletés de manipulation d'outils informatiques afin de tracer un parcours sur une carte numérique et de transmettre ce parcours à l'orienteur et à l'enseignant (rôle de traceur);

- mobiliser - ou acquérir via les ressources mises à leur disposition par l'enseignant - des habiletés de lecture de carte afin de prendre les photos à l'endroit exact des postes marqués sur la carte (rôles de poseur et d'orienteur);

- adopter un comportement fair-play en (a) dissociant et exécutant dans l'ordre demandé les tâches de traceur et de poseur ${ }^{3}$, (b) enregistrant et transmettant correctement les temps de course (rôles de poseur et d'orienteur), et (c) n'ayant recours à aucun instrument de navigation autre que la carte (rôles de poseur et d'orienteur).

6 - Formation et profession 28(4 hors-série), 2020 
En corollaire, cette analyse a priori permet d'anticiper des difficultés potentielles (a) chez les élèves en matière d'équipement et d'habiletés numériques, de gestion de l'autonomie, de la collaboration et de la communication au sein de la dyade, d'habiletés de lecture de carte et de gestion de course, et (b) chez les enseignants en matière d'enseignement à distance des habiletés numériques et de lecture de carte et d'engagement de tous les élèves dans l'activité.

\section{L'expérimentation}

Durant la période aiguë de la pandémie, la recherche dans les écoles romandes a été suspendue. Par conséquent, l'expérimentation a dû être menée auprès d'un public différent du public scolaire auquel la proposition curriculaire en $\mathrm{CO}$ était destinée. Cette dernière a ainsi été expérimentée par des étudiants $(n=22)$ qui étaient tenus de suivre un module "Didactique du Plein Air» dans le cadre de leur formation à l'enseignement de l'EP au secondaire dans une haute école pédagogique romande. Les consignes que ces derniers ont reçues de leurs formateurs sont reproduites en annexe 1. Elles précisent la proposition curriculaire originale en termes de durée/longueur du parcours et de nombre de postes, prescrivent l'usage de la boussole et préconisent le recours au site Web de cartographie de la Confédération helvétique (map.geo.admin) pour le traçage des parcours ainsi que l'utilisation d'une application de tracking GPS (bergfex) pour le contrôle et la validation par les formateurs du travail réalisé par les étudiants. Dès réception de ces consignes, les étudiants, par l'entremise d'un représentant, ont adressé aux formateurs une demande d'adaptation des tâches tenant compte (a) de la surcharge de travail occasionnée par les cours à suivre à distance, les nombreux travaux à rendre et la continuité pédagogique à assurer dans le cadre de leurs stages, et (b) des contraintes logistiques pour les étudiants dont la plupart résident dans des lieux bien plus éloignés géographiquement que ce n'est habituellement le cas des élèves d'une classe du secondaire I. En réponse à cette demande, les formateurs ont pris la décision de ne maintenir que la première partie du travail pratique de $\mathrm{CO}$, en l'occurrence la tâche de traceur-poseur, tout en invitant les étudiants qui le pouvaient à réaliser l'ensemble de la séquence didactique. Sur les 22 étudiants, deux ont constitué une dyade et réalisé l'ensemble de la séquence didactique, 19 ont réalisé la tâche de traceur-poseur et 1 n'a réalisé que la tâche de traceur, ayant été dispensé de la tâche de poseur pour raison médicale.

Les données recueillies lors de l'expérimentation sont constituées des cartes des parcours et des traces GPS respectives des étudiants. Elles ont été complétées par les réponses des étudiants (19/22) à un questionnaire en ligne qui leur a été transmis à l'issue de l'expérimentation. Celui-ci portait, en établissant un contraste entre la séquence originale et la séquence adaptée, sur (a) leur intérêt, (b) leur faisabilité, (c) leur transférabilité vers l'école et/ou l'après-pandémie, et (d) les bénéfices à en retirer. Il comportait (a) des questions fermées avec échelle de Likert en quatre points et (b) des zones de texte libre pour justifier ou commenter les réponses aux questions fermées.

\section{L'analyse a posteriori et la confrontation à l'analyse a priori}

Les documents transmis aux formateurs nous renseignent quant aux habiletés de manipulation d'outils informatiques et de lecture de carte mobilisées par les étudiants. La grande majorité des cartes numériques que ces derniers ont réalisées comportent le tracé d'un parcours sur lequel les postes sont représentés à l'aide de points (exemple en annexe 2). Sur les cartes tracées par les deux étudiants qui 
ont constitué une dyade et réalisé la séquence originale, seuls les postes sont symbolisés par des points (exemple en annexe 3). Enfin, un étudiant a fait figurer sur sa carte le tracé du parcours, la localisation des postes et les photos correspondant à chaque poste (annexe 4). Au vu de la qualité de ces tracés, nous pouvons considérer que le site Web de cartographie de la Confédération helvétique, préconisé dans les consignes, a constitué une ressource appréciable pour les étudiants. Le commentaire suivant issu du questionnaire illustre ce constat: « découverte du site map. geo. admin et de cette superbe fonctionnalité pour tracer des cartes et mesurer des distances ». Les parcours effectués par les traceursposeurs ont une longueur moyenne de 6,01 km (min. : 3,87; max. : 8,03) et comportent une moyenne de 10,24 postes (min. : 5; max. : 15), en accord avec les consignes reçues. Ils ont été parcourus à une vitesse moyenne de 8,79 km/h (min. : 5,00; max. : 12,25). Les traces GPS indiquent que les étudiants ont majoritairement eu recours à l'application bergfex préconisée dans les consignes.

De manière générale, les étudiants ne semblent donc pas avoir rencontré de difficultés, ni en matière d'équipement et d'habiletés numériques, ni en matière d'habiletés de lecture de carte et de gestion de course ${ }^{4}$. Les données quantitatives du questionnaire confirment ce constat. En effet, 89,47\% des répondants affirment que la tâche modifiée n'a pas posé de problème de faisabilité. Une majorité des répondants est même plutôt d'accord (38,89\%) ou tout à fait d'accord (33,33 \%) que telle que proposée et compte tenu du contexte, la tâche initiale aurait été faisable. Les commentaires fournis en complément aux questions fermées apportent une vision un peu plus nuancée : «la tâche ne m’a pas posé problème, l'application fonctionnait très bien et le lieu était facile d'accès "; « travail intéressant permettant d'utiliser de façon optimale les MITIC sans en abuser »; "il aurait fallu penser que certains étudiants ne pouvaient pas installer l'application demandée soit par manque de place soit car l'élève n'a pas de données cellulaires "; « les applications de traçage ont posé problème avec mes outils numériques »; « [difficulté] de transfert des données de mon parcours aux formateurs. Pas certain de la manière la plus adéquate de le faire mais un PDF et quelques captures d'écran avec explications n'est pas compliqué à faire "; « sous quel format envoyer les données serait à préciser. Utilisation de montre GPS [...] est plus simple mais pas forcément possible avec tout le monde ».

Cette tâche n'a pas seulement demandé aux étudiants de mobiliser des habiletés existantes, mais elle a suscité de véritables apprentissages, essentiellement dans le domaine de la formation générale et des capacités transversales, ainsi qu'en témoignent les réponses suivantes apportées par les étudiants à la question des bénéfices retirés par la réalisation de cette tâche : "grâce à cette tâche, on a pu apprendre à créer nos propres cartes. On est libre de créer nos cartes et nos itinéraires "; " apprendre à dessiner une carte (postes), faire une reconnaissance des lieux en amont »; « apprendre à utiliser plusieurs outils en même temps, savoir chercher des cartes sur Maps avec des tailles précises "; " maîtrise MITIC, investissement personnel, créativité »; "autonomie, approche ludique de la course d'endurance, collaboration avec un collègue ».

L'expérimentation ayant été menée auprès d'un public différent du public scolaire auquel la proposition curriculaire en CO était destinée, nous avons sollicité l'avis des étudiants sur la transférabilité de la séquence didactique originale vers des élèves en fin de cycle 3 de la scolarité obligatoire. Un bon tiers des répondants $(36,84 \%)$ ont jugé quelle était transférable telle quelle. Les autres $(63,16 \%)$ ont considéré qu'elle l'était moyennant différents types d'adaptation : parcours (plus court, moins de postes, 
périmètre délimité, course en étoile); organisation (transport vers le lieu de la $\mathrm{CO}$, création des parcours en binôme, former les binômes au préalable, possibilité de faire par groupe de quatre). Interrogés sur les bénéfices que des élèves en fin de cycle 3 pourraient retirer de cette séquence didactique, les étudiants ont mentionné des bénéfices similaires à ceux qu'ils avaient estimé avoir retirés eux-mêmes dans le domaine de la formation générale et des capacités transversales (autonomie, responsabilisation, prise de décision, collaboration, esprit de groupe, entraide, créativité, maîtrise MITIC, dépassement de soi). Ils ont cependant pointé des apprentissages plus spécifiques à la $\mathrm{CO}:$ « se repérer à l'aide d'une carte, tactique "; " découverte de la forêt et apprendre à lire une carte autre qu'une carte urbaine »; " lecture de carte, sur le terrain mais aussi pour créer le parcours et trouver des points repères intelligents, se repérer dans son environnement, "utilisation d'une boussole" »; « réaliser un tracé, se rendre compte des distances, lire une carte, course avec objectif ». Un répondant a en outre évoqué un bénéfice potentiel en matière d'engagement des élèves dans la tâche : « une meilleure implication des élèves dans la $\mathrm{CO}$ car ils auront été impliqués dans la réalisation de la tâche ». Un autre a déclaré avoir proposé la tâche modifiée à ses élèves en toute fin d'année scolaire, sans avoir de retour sur cette expérimentation non contrôlée : «j'ai repris cette course et l'ai proposée comme ultime activité. Les élèves pouvaient refaire mon parcours ou en créer un. Je n'ai pas eu de retour ».

\section{Discussion}

Dans cette contribution, nous rendons compte d'une démarche d'IDD qui avait pour visée de contribuer à la continuité pédagogique en EP, dans le contexte particulier de la pandémie COVID-19. Par rapport à d'autres types de recherche basés sur des expérimentations en classe, la méthodologie d'ingénierie se caractérise par le registre dans lequel elle se situe et les modes de validation qu'elle adopte (Artigue, 1988) :

En effet, les recherches ayant recours à des expérimentations en classe se situent le plus souvent dans une approche comparative avec validation externe basée sur la comparaison statistique des performances de groupes expérimentaux et de groupes témoins. Ce paradigme n'est pas celui de l'ingénierie didactique qui se situe, à l'opposé, dans le registre des études de cas dont la validation est essentiellement interne, fondée sur la confrontation entre analyse a priori et analyse a posteriori (p. 286).

Dans la présente étude de cas, le rôle déterminant des contraintes de la pandémie et du semi-confinement que celle-ci a provoqué apparaît clairement dans les analyses préalables et la conception d'une séquence didactique en EP, à commencer par le choix des pratiques sociales de référence (Martinand, 2001) qui se voyait réduit à de rares pratiques d'entretien (yoga, CrossFit), artistiques (danse) et de plein air $(\mathrm{CO})^{5}$, pour ne citer que celles qui ont été traitées par le groupe de recherche en didactique de l'EP de l'Université de X. Par ailleurs, ces contraintes ont doublement pesé sur l'expérimentation de cette séquence. Premièrement, les concepteurs de l'IDD ont dû mener celle-ci auprès d'un public différent du public scolaire auquel la proposition curriculaire en CO était destinée. Deuxièmement, les formateurs participant à cette expérimentation ont dû se résoudre à adapter la séquence originale à la demande de leurs étudiants. Cela n'est évidemment pas sans conséquences sur la validation interne de l'IDD, i.e. la confrontation entre analyse a priori et analyse a posteriori, et il nous semble important de discuter plus avant de ces conséquences. 
Menée en référence à la séquence originale et à un public scolaire en fin de cycle 3 de la scolarité obligatoire, l'analyse a priori a fait émerger un certain nombre de difficultés que les enseignants et les élèves étaient susceptibles de rencontrer dans l'enseignement/apprentissage de cette séquence. L'expérimentation menée auprès de formateurs et d'étudiants de l'enseignement supérieur pédagogique et l'analyse a posteriori n’ont pas confirmé celles-ci. Ainsi, aucune difficulté majeure n'a été relevée chez les étudiants en matière d'équipement et d'habiletés numériques. Il est vrai que les formateurs ont fait preuve d'ingéniosité didactique (Schubauer-Leoni et Dolz, 2004) en préconisant aux étudiants l'usage d'applications de cartographie et de tracking GPS performantes, que ces derniers ont pu s'approprier de manière autonome. Il n'en aurait probablement pas été de même avec des élèves du secondaire I. En effet, la représentation commune que les digital natives auraient de la facilité à acquérir les habiletés numériques constitutives de ce qu'il est désormais convenu d'appeler la littératie numérique a été contredite par des études récentes (Schmid et Petko, 2019; Šorgo, Bartol, Dolničar et Boh Podgornik, 2017). Par ailleurs, si les adolescents des années 2000 se différencient de moins en moins par la possession et la fréquence d'utilisation d'un ordinateur, ils continuent en revanche à se différencier des adolescents issus des milieux favorisés qui entretiennent une plus grande familiarité avec les usages connectés (Collin, Guichon et Ntébutsé, 2015; Mercklé et Octobre, 2012). Par conséquent, dans une classe du secondaire I plus mixte socialement, il est probable que certains élèves auraient éprouvé des difficultés à accomplir de manière autonome les activités médiatiques de production (Fastrez et De Smedt, 2012) incluses dans la séquence didactique. De même, aucune difficulté majeure n’a été relevée chez les étudiants en matière d'habiletés de lecture de carte et de gestion de course, tous ayant déjà bénéficié d'un enseignement de $\mathrm{CO}$ dans leur formation disciplinaire antérieure. La CO n'apparaissant qu'au cycle 3 dans le PER parmi les APSA susceptibles d'être enseignées durant la scolarité obligatoire, il est probable que des élèves de ce cycle auraient eu besoin de recevoir un enseignement à distance sur ces contenus, et en corollaire, que des enseignants de ce degré scolaire auraient pu éprouver des difficultés à prodiguer ce type d'enseignement à distance.

D’un autre côté, il est bien connu que l'ingénierie échappe partiellement à ses concepteurs au moment de l'expérimentation, les enseignants pouvant prendre des initiatives imprévues qui perturbent le fonctionnement du processus de validation (Artigue et Perrin-Glorian, 1991). Ces distorsions s'accentuent lorsque l'on quitte le cadre contrôlé de la recherche, ce qui est le cas ici en raison des contraintes de la pandémie. La comparaison entre la séquence didactique originale Tableau 2) et les consignes des formateurs à leurs étudiants (Annexe 1) témoigne néanmoins de la bonne appropriation $\mathrm{du}$ produit de l'IDD par ses utilisateurs. Nous n'avons en revanche aucun retour d'éventuels enseignants du cycle 3 qui auraient mis cette séquence à l'épreuve, et ne pouvons par conséquent nous prononcer sur son potentiel de reproductibilité interne (Artigue et Perrin-Glorian, 1991).

Finalement, les contraintes logistiques ayant amené les étudiants à demander à leurs formateurs une adaptation de la séquence originale n'avaient pas été anticipées par les concepteurs de l'IDD. Dans le questionnaire, les étudiants ont toutefois relativisé ces contraintes et leur impact sur la faisabilité par des élèves plus jeunes de la $\mathrm{CO}$ sous forme de duel en dyades, à l'exemple de ce commentaire qui soulignait en outre le plus grand intérêt de la séquence originale par rapport à sa version simplifiée : « la CO en binôme est plus complète et a plus de sens, mais il faut que les "élèves" soient proches. En secondaire I, ceci ne devrait pas poser de problème, en secondaire II, les situations seraient très variées ». 


\section{Conclusion}

La description livrée ici des quatre phases d'une IDD visant à contribuer à la continuité pédagogique en EP montre le double effet de la pandémie COVID-19 et de ses contraintes sur la démarche d'ingénierie. Premièrement, un effet de catalyseur a poussé les concepteurs de l'IDD à identifier les APSA et les contenus d'enseignement les plus à même d'être transmis et acquis à distance, et à faire appel de façon raisonnée aux MITIC pour maintenir possible la communication des élèves avec l'enseignant et leurs pairs. Il en a résulté des séquences didactiques préservant les enjeux d'apprentissage et de transmission culturelle de l'EP, à l'image de la séquence en CO analysée ici. Nous pensons que ce travail de conception sous contrainte peut constituer une source d'inspiration pour les enseignants, afin de développer les formes d'enseignement hybrides qui se profilent inexorablement comme l'avenir de l'EP, pas seulement en période de pandémie, tout en évitant les écueils de la médicalisation et de la marchandisation de l'EP qui pèsent sur le futur de cette discipline scolaire (Gard, 2014; Williamson, 2015). Deuxièmement, un effet d'obstacle restreint les opportunités d'accès au terrain scolaire pour expérimenter et valider les séquences didactiques conçues dans les premières phases de l'IDD. Il ne s'agit pas d'une fatalité et il conviendrait selon nous d'élaborer des protocoles d'IDD mieux adaptés aux contraintes de la pandémie, au cas où de nouveaux épisodes devaient se reproduire à l'avenir.

\section{Notes}

1 Dans le PER, les attentes dites fondamentales désignent les apprentissages que chaque élève devrait atteindre au cours, mais au plus tard à la fin du cycle.

2 Cette seconde partie de la tâche du traceur-poseur correspond à la tâche dite des "poseurs-contrôleurs " (Issaulan et Lamotte, 2005, cité par Mottet et Saury, 2014). Selon ces derniers auteurs, « le pari didactique sous-jacent à la conception de cette tâche est que ses contraintes favorisent une activité de navigation plus fine et sollicitent plus fortement la capacité de lecture précise de leur environnement» (p. 42).

3 En effet, lélève traceur-poseur qui réaliserait le parcours et prendrait des photos au cours de celui-ci avant de reporter les postes correspondants sur la carte pourrait se procurer un avantage chronométrique par rapport à lélève qui réaliserait la tâche du traceur-poseur dans les conditions demandées.

4 La faible performance d'une étudiante ayant réalisé son parcours à la vitesse moyenne de $5 \mathrm{~km} / \mathrm{h}$ n'est pas due à des difficultés particulières en matière d'habiletés de lecture de carte ou de gestion de course. Elle s'explique par des raisons de santé, ainsi que cette étudiante l'a signalé dans les documents transmis aux formateurs : "J'ai effectué une course d'orientation de $5.52 \mathrm{~km}$ car avec mon genou je n'arrive pas encore à courir sur une longue durée et sur un sol variable. J'ai principalement marché c'est pour cela que j'ai dépassé les 45 minutes ».

5 À noter que l'enseignement à distance de ces pratiques de plein air était possible en Suisse, mais ne l'aurait pas été dans d'autres pays appliquant un confinement plus strict, tels que la France par exemple qui interdisait les déplacements non essentiels au-delà d'un rayon de $1 \mathrm{~km}$ autour du domicile.

\section{Références}

Amade-Escot, C. et Marsenach, J. (1995). Didactique de l'éducation physique et sportive. Questions théoriques et méthodologiques. Grenoble : La Pensée Sauvage.

Artigue, M. (1988). Ingénierie didactique. Recherches en Didactique des Mathématiques, 9(3), 281-308.

Artigue, M. et Perrin-Glorian, M.-J. (1991). Didactic engineering, research and development tool : Some theoretical problems linked to this duality. For the Learning of Mathematics, 11(1), 13-18. 
Carnus, M.-F., Sauvegrain, J.-P. et Terrisse, A. (2002). Intérêt et utilisation de la méthodologie d'ingénierie didactique dans l'analyse du processus décisionnel de l'enseignant d'EPS. Les Dossiers des Sciences de l'Éducation, 8(1), 99-112.

Charnay, R. (2003). L'analyse a priori, un outil pour l'enseignant. Maths-École, 209, 19-26.

Collin, S., Guichon, N. et Ntébutsé, J.-G. (2015). Une approche sociocritique des usages numériques en éducation. Sciences et Technologies de l'Information et de la Communication pour l'Éducation et la Formation, 22(1), 89-117.

Conférence Intercantonale de l'Instruction Publique de la Suisse Romande et du Tessin (2010-2016). Plan d'études romand. Repéré le 30 juin 2020 à https://www.plandetudes.ch.

Fastrez, P. et De Smedt, T. (2012). Une description matricielle des compétences en littératie médiatique. Dans M. LebrunBossard, N. Lacelle et J.-F. Boutin (dir.), la littératie médiatique multimodale. De nouvelles approches en lecture-écriture à l'école et hors de l'école (p. 45-60). Québec : PUQ.

Forest, E., Lenzen, B. et Öhman, M. (2018). Teaching traditions in physical education in France, Switzerland and Sweden: A special focus on official curricula for gymnastics and fitness training. European Educational Research Journal, 17(1), 71-90.

Gard, M. (2014). eHPE: a history of the future. Sport, Education and Society, 19(6), 827-845.

Jourand, C., Adé, D., Sève, C., Komar, J. et Thouvarecq, R. (2018). Dynamics of student interactions: an empirical study of orienteering lessons in physical education. Physical Education and Sport Pedagogy, 23(2), 134-149.

Jourand, C., Adé, D., Sève, C. et Thouvarecq, R. (2016). Matérialité et formes d'interaction entre élèves : une étude empirique lors de leçons d'EPS. eJRIEPS, 37, 30-57.

Marsenach, J. et Amade-Escot, C. (1993). Les orientations de la recherche en didactique de l'éducation physique et sportive. Revue française de pédagogie, 103, 33-41.

Martinand, J.-L. (2001). Pratiques de référence et problématique de la référence curriculaire. Dans A. Terrisse (dir.), Didactique des disciplines. Les références au savoir (pp. 17-24). Bruxelles : De Boeck.

Mercklé, P. et Octobre, S. (2012). La stratification sociale des pratiques numériques des adolescents. RESET [en ligne]. Repéré le 13 juillet 2020 à http://journals.openedition.org/reset/129.

Mottet, M. et Saury, J. (2014). Analyse compréhensive de l'activité de navigation d'orienteurs débutants en fonction des caractéristiques de deux tâches de course d'orientation. STAPS, 104(2), 39-55.

Perrenoud, P. (1998). La transposition didactique à partir de pratiques : des savoirs aux compétences. Revue des sciences de l'éducation, 24(3), 487-514.

Perrin-Glorian, M.-J. (2011). L'ingénierie didactique à l'interface de la recherche avec l'enseignement. Développement de ressources et formation des enseignants. Dans C. Margolinas, M. Abboud-Blanchard, L. Bueno-Ravel et N. Douek (dir.), En amont et en aval des ingénieries didactiques (pp. 57-78). Grenoble : La Pensée Sauvage.

Perrin-Glorian, M.-J. (2019). A l'interface entre recherche et enseignement, les ingénieries didactiques. Actes du congrès : La TACD en questions, questions à la didactique (Vol. 4,pp. 115-127). Rennes : CREAD. Repéré le 25 juin 2020 à https://tacd-2019.sciencesconf.org/data/ACTES Session4 Congres TACD Rennes 2019.pdf.

Schmid, R. et Petko, D. (2019). Does the use of educational technology in personalized learning environments correlate with self-reported digital skills and beliefs of secondary-school students? Computers E Education, 136, 75-86s

Schubauer-Leoni, M.-L. et Dolz, J. (2004). Comprendre l'action et l'ingéniosité didactique de l'enseignant : Une composante essentielle de la transformation de l'École. Dans J.-P. Bronckart et M. Gather-Thurler (dir.), Transformer l'école (pp. 147-168). Bruxelles : De Boeck.

Šorgo, A., Bartol, T., Dolničar, D. et Boh Podgornik, B. (2017). Attributes of digital natives as predictors of information literacy in higher education. British Journal of Educational Technology, 48(3), 749-767.

Swiss Orienteering (2009-2010). La course d'orientation. Le sport à valeur ajoutée. Repéré le 15 mars 2020 à https://www.swiss-orienteering.ch/files/broschueren/ol fr.pdf.

Travert, M. et Mascret, N. (dir.) (2011). La culture sportive. Paris : Éditions EP\&S. 
Intégrer les contraintes de la pandémie dans les analyses préalables, la conception et l'expérimentation d'ingénieries didactiques en éducation physique. Un exemple en course d'orientation

Williamson, B. (2015). Algorithmic skin: health-tracking technologies, personal analytics and the biopedagogies of digitized health and physical education. Sport, Education and Society, 20(1), 133-151.

\section{Pour citer cet article}

Lenzen, B., Deriaz, D. et Voisard, N. (2020). Intégrer les contraintes de la pandémie dans les analyses préalables, la conception et l'expérimentation d'ingénieries didactiques en éducation physique. Un exemple en course d'orientation. Formation et profession, 28(4 hors-série), 1-17. http://dx.doi.org/10.18162/fp.2020.677 


\section{Annexe 1}

\section{Consignes aux étudiants pour l'expérimentation}

\section{Thématique 1 : course d'orientation (CO)}

\section{Objectifs}

1) Préparer une $\mathrm{CO}$ pour un·e collègue du cours de didactique

2) Tester et chonométrer la CO préparée par soi-même

3) Réaliser la CO préparée par un·e collègue du cours de didactique

\section{Consignes}

$\square$ Se rendre sur le site de l'équipe de $X$ de l'Université de $X$ pour prendre connaissance du projet de CO.

C Constituer un binôme avec un.e collègue de classe proche de son domicile.

0 Préparer un parcours de $\mathrm{CO}$ en tenant compte des modalités indiquées dans le projet précité et des consignes ci-dessous.

Etape 1 : préparation du parcours

Se rendre sur le site de la Confédération map.geo.admin

0 Choisir la zone de la $\mathrm{CO}$ : à votre convenance, si vous le souhaitez en concertation avec votre binôme (idéalement en plein nature, possible aussi en zone urbaine ou périurbaine)

- Sous le menu " Dessiner et mesurer » dessiner votre parcours puis l'imprimer à l'échelle $1: 10000$ (identique à la CO). Ajouter les postes (points de repères) et si besoin un commentaire (un indice). Les postes ne sont pas posés à l'avance. II s'agit de points de repère bien visibles et remarquables lorsqu'on se trouve au bon endroit.

(- Imprimer votre carte au format A4.

0 Critères à respecter :

○ durée : environ $45 \mathrm{~min}$ (soit environ $7 \mathrm{~km}$, selon dénivelé)

○ nombre de « postes » : environ 10.

( Sur votre smartphone, installer une application de tracking GPS. Nous vous conseillons d'utiliser l'application bergfex, application gratuite sur Google Play ou Apple Store.

Etape 2 : reconnaissance et course

Se rendre sur le terrain muni de la carte, d'une boussole (si possible analogique) et de votre smartphone.

E Effectuer le parcours, prendre les photos (selfie ou non) des postes correspondants en enregistrant la trace GPS et les données numériques de votre course (durée, vitesse moyenne, etc.), ce que fait très bien l'application bergfex.

Enregistrer cette trace sur votre téléphone.

Etape 3 : envoyer votre parcours à votre binôme.

Etape 4 : effectuer le parcours que vous a préparé votre binôme, ici aussi en enregistrant les données de votre course.

Etape 5 : organisez une visioconférence avec votre binôme, comparez vos résultats (qui a gagné ?) et échangez sur les divers aspects de cette expérience.

Etape 6 : transmettez-nous par email la carte de votre parcours au format PDF ainsi que la trace GPS de vos deux courses (la vôtre et celle préparée par votre partenaire). 


\section{Annexe 2}

Exemple de carte topographique numérique avec tracé du parcours et postes générée via la plateforme map.geo.admin de l'Office fédéral de la cartographie de la Confédération helvétique

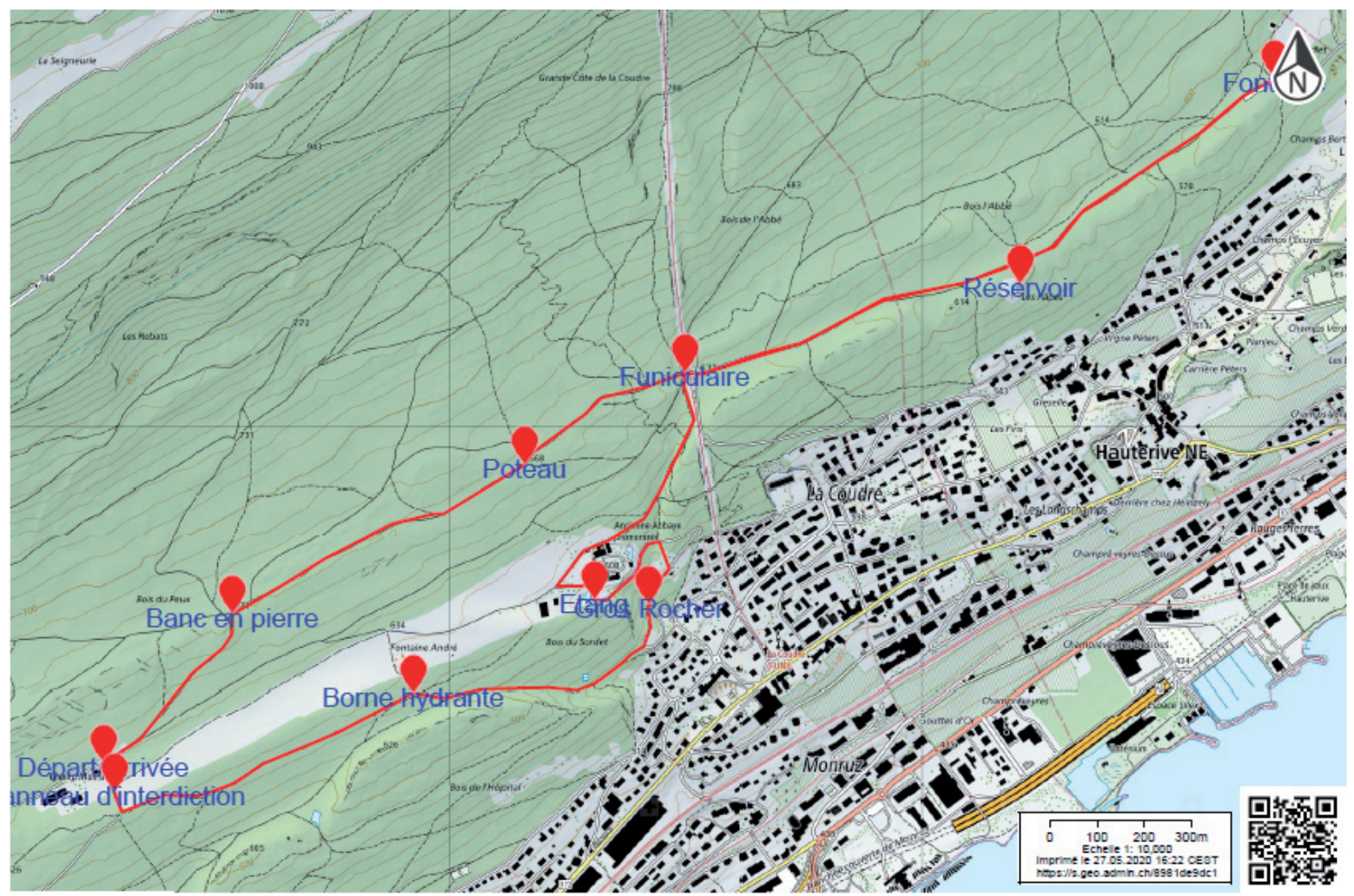




\section{Annexe 3}

Exemple de plan satellite avec postes

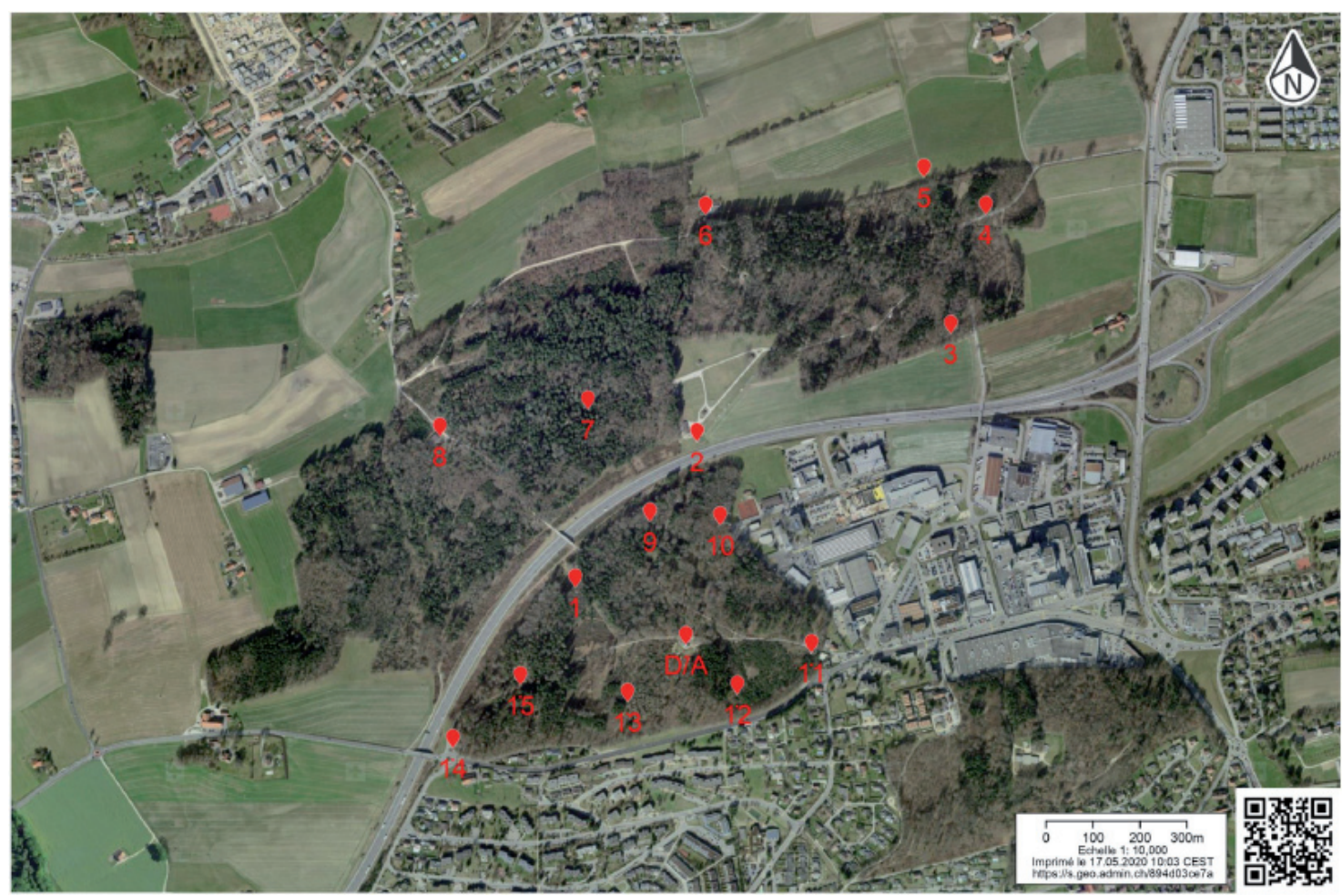




\section{Annexe 4}

Carte avec tracé du parcours, postes et photos

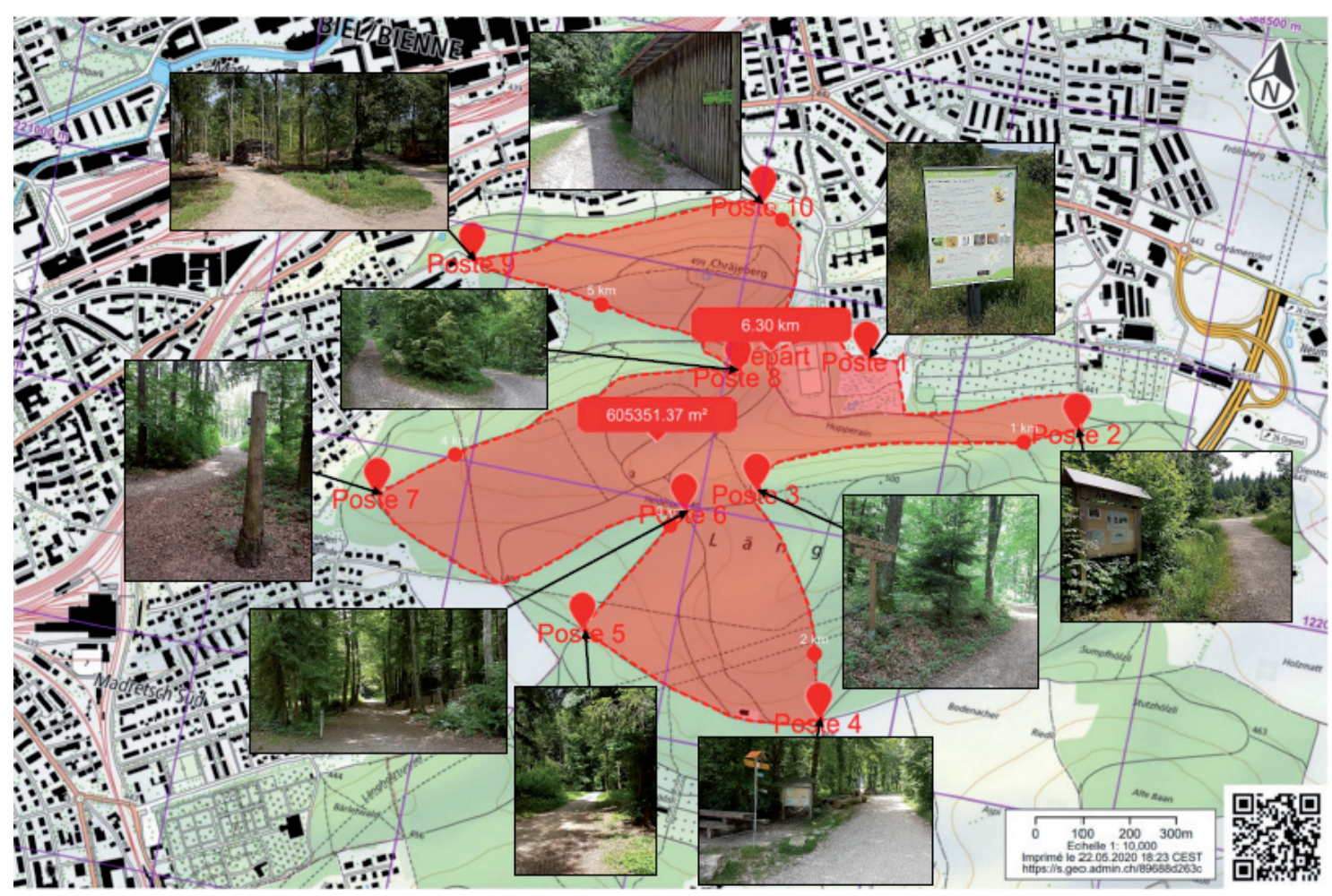

